\title{
On the soliton width in the incommensurate phase of spin-Peierls systems.
}

\author{
Ariel Dobry and José A. Riera \\ Departamento de Física, Universidad Nacional de Rosario, \\ and Instituto de Física Rosario, Avenida Pellegrini 250, 2000 Rosario, Argentina
}

\begin{abstract}
We study using bosonization techniques the effects of frustration due to competing interactions and of the interchain elastic couplings on the soliton width and soliton structure in spin-Peierls systems. We compare the predictions of this study with numerical results obtained by exact diagonalization of finite chains. We conclude that frustration produces in general a reduction of the soliton width while the interchain elastic coupling increases it. We discuss these results in connection with recent measurements of the soliton width in the incommensurate phase of $\mathrm{CuGeO}_{3}$.

PACS numbers: 75.10-b,75.10Jm,75.Kz,75.80.+q
\end{abstract}

One dimensional (1D) magnetic systems are unstable against a spin-lattice interaction. At a finite temperature a phase transition takes place toward a dimerized or spin-Peierls (SP) state whose magnetic signature is the opening of a gap (SP gap) between the singlet ground state and triplet excitations. The recent discoveryl of the first inorganic SP compound $\mathrm{CuGeO}_{3}$ has renewed the interest in these quasi-one-dimensional spin-phonon coupled systems. A number of experiments can be performed on these inorganic materials in an easier way than on the organic SP systems. It is also now possible to replace the $\mathrm{Cu}$ ions by other magnetic or nonpagnetic ions and to study the effects of these impurities 2 Adding interest to the study of this compound is the fact that, as it was early recognized, the temperature dependence of the spin susceptibility could only be accounted for if an important next nearest-neighbor (NNN) is included in the 1D Heisenberg model used to describe this material H $_{\text {t }}$ The dispersion of magnetic excitations and a number of other experimentally determined properties are also consistent with a model with NNN interaction.

Recent x-ray-scattering experiments have detected a transition from the uniform SP phase into an incommensurate phase 8 in the presence of a magnetic field. The experimental x-ray spectra in the incommensurate state has been interpreted as due to a soliton lattice structure. A soliton deformation was early theoretically predicted for SP systemse. It arises because the Zeeman energy favors states with nonzero magnetic moment thus breaking the dimer pattern. That is, each down spin changed into an up spin breaks a singlet dimer and gives rise to two solitons, each soliton carrying a spin- $\frac{1}{2}$.

The soliton width has been experimentally estimated from the relationship between the measured intensities of the main x-ray peak and its harmonics. 0 The resulting value is $\xi=13.6 c$ with $c$ the lattice constant in the spin chain direction ( $c=1$ hereafter). The predicted width for a one-dimensional model with only nearest neighbor (NN) interaction is given by $\xi=J \pi /(2 \Delta)$, where $\mathrm{J}$ is the exchange coupling and $\Delta$ the SP gap. 9 Using the experimental value of $\Delta=2.1 \mathrm{meV}$ and $J=120 \mathrm{~K}$, estimated by fitting the spin susceptibility with a Heisenberg model with NN interactions, the predicted $\xi$ is equal to 8 which is considerably smaller than the experimental one.

It is then quite apparent that some other ingredients should be added to the model in order to explain the origin of this disagreement between theory and experiment. One of the goals of the present work is to analize the effect of the frustration induced by the competing $\mathrm{NN}$ and NNN antiferromagnetic interactions on the soliton width. In this sense, we are going to extend the formalism which leads to the relationship between the soliton width and the gap, originally developed in Ref. [9].

Another possible contribution to the soliton width comes from the interchain elastic coupling. It is well known that the three-dimensional (3D) character of the phonon field is essential to account for the finite temperature SP transition. Moreover, for the specific case of $\mathrm{CuGeO}_{3}$, it has been found that the principal lattice anomaly takes place in a direction perpendicular to the spin chain 10.11 and it is related with displacements of the oxygen ions toward and against the chains. In this situation the phonon modes perpendicular to the chains involving coherent motion of the atoms in different chains become relevant in the SP phase. 10 It is then reasonable to assume that the interchain elastic coupling could be relevant to explain some features of the system. The possibility that interchain elastic coupling could modify the soliton shape is supported by a recent measurement of the elastic constants in $\mathrm{CuGeO}_{3}$ in the presence of high magnetic fields. 12 In this study, it was shown that the elastic constant along the $\mathrm{b}$ axis is the one that presents the largest variation at the dimerized-incommensurate transition.

We conclude that frustration produces in general a reduction of the soliton width while the elastic coupling of a chain with its neighbors increases it.

We start our study by analyzing the effects of frustration on the soliton width. Therefore, we consider the following 1D model Hamiltonian:

$$
H=H_{e l}+H_{m g}
$$




$$
\begin{gathered}
\frac{H_{e l}}{J}=\frac{1}{2} K_{\|} \sum_{i}\left(u_{i+1}-u_{i}\right)^{2} \\
\frac{H_{m g}}{J}=\sum_{i}\left\{\left(1+\left(u_{i+1}-u_{i}\right)\right) \mathbf{S}_{i} \cdot \mathbf{S}_{i+1}+\alpha \mathbf{S}_{i} \cdot \mathbf{S}_{i+2}\right\}
\end{gathered}
$$

where $\mathbf{S}_{\mathbf{i}}$ are spin-1/2 operators and $u_{i}$ is the displacement of magnetic ion $i$ from its equilibrium position. Notice that in $\mathrm{CuGeO}_{3}$, the displacements $u_{i}$ are related to the displacements of the $\mathrm{O}$ ions. $H_{e l}$ in Eq. (1) corresponds to the elastic energy interaction of the ions along the chain with dimensionless elastic constant $K_{\|}$. We include the phonons in the adiabatic approximation and we allow arbitrary displacement patterns.

We now go to the bosonic representation of Hamiltonian (1). This is achieved by first applying a JordanWigner transformation on the spin operators and then linearizing the resulting fermionic relation dispersion around the Fermi level. Finally, the fermionic field theory is bosonized. Very precise predictions have been obtained within this approach for the ground state and -low energy excitations of gne-dimensional spin systems 13 . Nakano and Fukuyama 9 used this bosonic representation to study soliton formation in the unfrustrated chain. In the following, we extend their approach to the frustrated chain. In terms of the boson field $\phi$ and its conjugate momentum $\Pi$ the Hamiltonian (11) can be rewritten as:14

$$
\begin{gathered}
H_{e l}=2 K_{\|} \int d x u^{2} \\
H_{b o s}=\int \frac{d x}{2 \pi}\left\{\frac{v_{s}}{\eta}\left(\partial_{x} \phi\right)^{2}+v_{s} \eta(\pi \Pi)^{2}+u \sin \phi\right. \\
-g \cos (2 \phi)\}
\end{gathered}
$$

In going from (11) to (2,3) we have replaced the lattice displacement $u_{i}$ by a continuum field as:

$$
(-1)^{i} u_{i} \rightarrow u(x)
$$

so that we can describe smooth deviations of the displacement field with respect to the dimerized pattern.

The quantities $v_{s}, \eta$ and $g$ are expressed in terms of the microscopic parameters of Hamiltonian (11). The expressions can be read in Ref. [14]. However, once identified the physical meaning of these quantities it is customafy to replace those expressions by more precise relations. 13 In this sense, the isotropy of the long distance behavior of the correlation functions implies that $\eta=2$. The parameter $g$ measures the strength of the umklapp processes in the fermionic theory. The last term of (3) is a marginally irrelevant operator for all $g>0$, so that in absence of coupling to the lattice a gapless spin liquid phase is predicted. When $\mathrm{g}$ is negative the last term of Eq. (3) becomes a relevant operator leading the system to a spontaneously dimerized gapped phase 13 Therefore, $g$ should be proportional to $\alpha_{c}-\alpha$ where $\alpha_{c}$ is the critical value separating the gapless and the gapped phases. The quantity $v_{s}$ is the spin wave velocity. For $\alpha=0$, $v_{s}=J \pi / 2$ from Bethe's exact solution. For $\alpha<\alpha_{c}, v_{s}$ has been recently numerically evaluated as the derivative of the magnon dispersion relation at the bottom of the band 16 This derivative is not well defined for $\alpha>\alpha_{c}$ due to the presence of the gap above discussed. We will analyze later on our procedure to evaluate $v_{s}$ in this case.

The application of a magnetic field to the system favors states with nonzero $S_{z}$ and to the appearance of solitons. Each soliton could be regarded as a domainwall separating two opposite dimerization patterns. To take into account these states we follow the approach of Ref. [9]. We separate the variable $\phi$ into the sum of a classical variable $\phi_{0}$ and its quantum fluctuation $\hat{\phi}$. A self-consistent harmonic approximation is then used. We retain $\hat{\phi}$ to quadratic order and we require the vanishing of the first order term. The displacement field $u$ is obtained from its static equilibrium equation. The resulting equations are:

$$
\begin{gathered}
-\frac{2 v_{s}}{\eta}\left(\partial_{x}^{2} \phi\right)+u e^{-\left\langle\hat{\phi}^{2}\right\rangle / 2} \cos \phi_{0}-2 g e^{-2\left\langle\hat{\phi}^{2}\right\rangle} \sin \left(2 \phi_{0}\right)=0 \\
\sin \phi_{0} e^{-\left\langle\hat{\phi}^{2}\right\rangle / 2}+4 K_{\|} u=0
\end{gathered}
$$

where $\left\langle\hat{\phi}^{2}\right\rangle$ is the ground state expectation value which we assume the same as in the dimerized phase. These equations could be combined to give:

$$
\left(\partial_{x}^{2} \phi_{0}\right)+\frac{1}{2 \xi^{2}} \sin \left(2 \phi_{0}\right)=0
$$

The soliton solution of this equation is:

$$
\sin \left(\phi_{0}\right)=\tanh (x / \xi)
$$

The lattice soliton is then given by:

$$
u(x)=u_{0} \tanh (x / \xi)
$$

with $u_{0}$ the equilibrium displacement in the uniform dimerized state. The main quantity we are interested in is the soliton width which is given by:

$$
\frac{1}{\xi^{2}}=\frac{\eta}{2 v_{s}}\left[\frac{e^{-<\hat{\phi}^{2}>/ 2}}{4 K_{\|}}+4 g e^{-2<\hat{\phi}^{2}>}\right] \text {. }
$$

This quantity is related to the singlet-triplet gap in the uniform dimerized state in a simple way. In this state $u(x)=u_{0}$ and $\phi_{0}=\pi / 2$. By following similar steps as in Ref. [9] we obtain the dispersion relation for the excitations of the system given by:

$$
\varepsilon(k)=v_{s}\left(k^{2}+k_{0}^{2}\right)^{1 / 2}
$$

where $k_{0}=1 / \xi$ is given by Eq. (9). Note that $v_{s}$ is a function of $\alpha$ in this case. Finally, the simple scaling relation between the soliton width and the $\mathrm{SP}$ gap $\Delta$, 


$$
\xi=v_{s} / \Delta
$$

originally obtained for the unfrustrated chain, is also valid in the presence of frustration. For $\alpha>\alpha_{c}, k_{0}$ contains a contribution from the frustration due to the presence of a gap even in the absence of dimerization.

The relation (11) can be tested by numerical exact diagonalization on finite chains. The adiabatic equations corresponding to Hamiltonian (11) are:

$$
K_{\|} \delta_{i}+\left\langle 0\left|\mathbf{S}_{i} \cdot \mathbf{S}_{i+1}\right| 0\right\rangle=0
$$

where $\delta_{i}=\left(u_{i+1}-u_{i}\right)$ is the bond length variable. We have solved iteratively these equations for $\delta_{i}$ starting from a random configuration $\left\{\delta_{i}^{(0)}\right\}$. The ground state of the spin system $|0\rangle$ was recalculated at each iteration step by Lanczos diagonalization. We considered finite chains of even number of sites up to 20 sites with periodic boundary conditions. The numerical details of the method are given elsewhere15.

In the subspace $S^{z}=0$ the system converges to a dimerized configuration $\left\{\delta_{i}^{(0)}\right\}$ as expected. For $\alpha=0.0$, 0.2 and 0.4 , and for several values of $K_{\|}$, using the dimerized pattern obtained at $S^{z}=0$, we computed the spin gap as $\Delta=E_{0}\left(S^{z}=1\right)-E_{0}\left(S^{z}=0\right)$.

When the spin Hamiltonian is diagonalized in the subspace $S^{z}=1$ we found that two solitons appear in the dimer pattern. The deformation pattern $\left\{\delta_{i}\right\}$ could be described by a superposition of two solitons centered at sites $i_{1}$ and $i_{2}$ of the form:

$$
\delta_{i}=(-1)^{i} \delta_{0} \tanh \left(\frac{i-i_{1}}{\xi}\right) \tanh \left(\frac{i-i_{2}}{\xi}\right)
$$

where $\delta_{0}$, the deformation amplitude, and $\xi$, the soliton width, were obtained by numerical fitting. The main limitation of this calculation arises in the region where, for a given $\alpha, K_{\|}$is so large that the solitons have a substantial overlap in our small chain and the fitting function (13) is no longer appropriate. Unfortunately, it is precisely in this situation where the continuum approximation leading to the relation (11) is expected to hold.

In Fig. 1 we show the soliton width $\xi$ as a function of $1 / \Delta$ for the same values of $\alpha$ as above. The linear behavior predicted by Eq. (111) is clearly seen. A linear fitting of these curves in the region $\xi>2.5$ gives the slopes 1.87, 1.70 and 1.63 , for $\alpha=0.0,0.2$ and 0.4 respectively. For $\alpha<\alpha_{c}$, a numerical calculation 16 leads to $v_{s}=\frac{\pi}{2}(1-1.12 \alpha)$ in the thermodynamic limit. From this law, $v_{s}=1.57,1.22$, for $\alpha=0.0$ and 0.2 respectively. We can observe that the slopes obtained by fitting the numerical data are larger than the calculated $v_{s}$. Besides, the effect of $\alpha$ is weaker in the numerical data than that predicted by Eq. (11). It is difficult to conclude at this point if this disagreement between the prediction obtained by the continuum bosonized theory and numerical results is due to the approximations involved in the former or to finite size effects. For $\alpha=0.4>\alpha_{c} \approx 0.2411$, we have estimated $v_{s}$ by fitting the energy $\varepsilon(k)^{2}=\Delta^{2}+v_{s}^{2} k^{2}+c k^{4}$ around $k=0$, where $\varepsilon(k)=E_{0}\left(S^{z}=1, k\right)-E_{0}\left(S^{z}=0, k=0\right)$. For $L=20$, we obtained $v_{s}=0.707$, a value which is smaller than the slope of the curve $\xi$ vs. $1 / \Delta$ for $\alpha=0.4$ in Fig. (11).

Then the qualitative picture of the effect of frustration is partially confirmed by the exact diagonalization study. Both analytically and numerically we have seen that frustration reduces the soliton width for a given value of the SP gap. However, the numerical calculations show a smaller dependence on $\alpha$ than that analytically predicted.

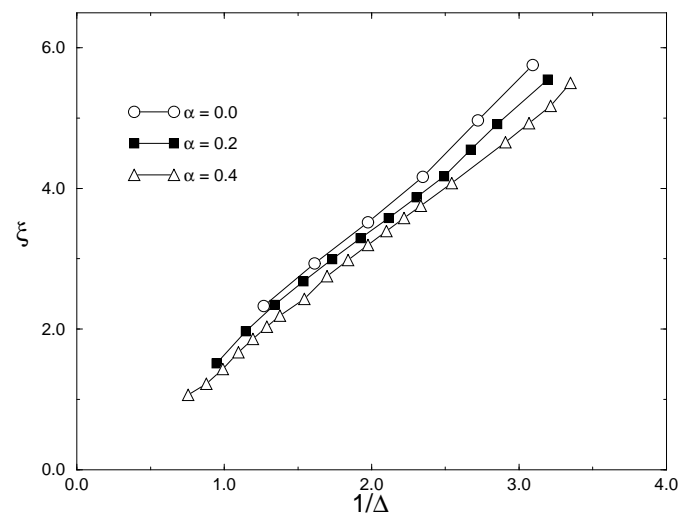

FIG. 1 . Soliton width vs. $\Delta^{-1}$ obtained by exact diagonalization in the 20 site chain for various values of $\alpha=J_{2} / J$.

We now turn to the study of the effects caused by the $3 \mathrm{D}$ character of the phonon field. As previously discussed, there are both theoretical as well experimental arguments indicating that the interchain elastic coupling is important to describe the excitations of the system in the SP regime. Therefore, we add to $H_{e l}$ of Eq. (11) an interchain elastic coupling of the form:

$$
\begin{aligned}
H_{e l}^{\prime}=\sum_{i, \mathbf{j}} & \left\{\frac{K_{\perp}^{a}}{2}\left(u_{i}^{j_{x}+1, j_{y}}-u_{i}^{\mathbf{j}}\right)^{2}\right. \\
& \left.+\frac{K_{\perp}^{b}}{2}\left(u_{i}^{j_{x}, j_{y}+1}-u_{i}^{\mathbf{j}}\right)^{2}\right\}
\end{aligned}
$$

where $u_{i}^{\mathbf{j}}$ denotes the displacement of the $\mathbf{j}$ ion in the chain $\mathbf{j}\left(\mathbf{j} \equiv\left(j_{x}, j_{y}\right)\right) . K_{\perp}^{a}$ and $K_{\perp}^{b}$ are the harmonic couplings in the $a$ and $b$ directions respectively. We assume that there is no interchain magnetic interactions. The system now consists of a set of spin chains immersed in a $3 \mathrm{D}$ lattice. We resort again to bosonization methods to analyze soliton formation in this system. The boson field $\phi$ turns to be $\mathbf{j}$-dependent. The adiabatic condition (5) is now given by:

$$
\begin{aligned}
& \sin \phi_{0}^{\mathbf{j}} e^{-<\hat{\phi}^{2}>/ 2}+\left[2\left(K_{\perp}^{a}+K_{\perp}^{b}\right)+4 K_{\|}\right] u^{\mathbf{j}}- \\
& K_{\perp}^{a}\left(u^{j_{x}+1, j_{y}}-u^{j_{x}-1, j_{y}}\right)-K_{\perp}^{b}\left(u^{j_{x}, j_{y}+1}-u^{j_{x}, j_{y}-1}\right)=0 .
\end{aligned}
$$

This equation can be inverted to give: 


$$
u^{\mathbf{j}}(x)=-\frac{1}{4 K_{\|}} \sum_{\mathbf{j}^{\prime}} \mathbf{B}\left(\mathbf{j}^{\prime}-\mathbf{j}\right) \sin \phi_{0}^{\mathbf{j}^{\prime}}(x) e^{-<\hat{\phi}^{2}>/ 2}
$$

where:

$$
\mathbf{B}\left(\mathbf{j}^{\prime}-\mathbf{j}\right)=\int_{-\pi}^{\pi} \int_{-\pi}^{\pi} \frac{d \mathbf{k}}{(2 \pi)^{2}} \frac{\cos \left[\mathbf{k} \cdot\left(\mathbf{j}^{\prime}-\mathbf{j}\right)\right]}{1+\epsilon^{a} \sin ^{2} \frac{k_{x}}{2}+\epsilon^{b} \sin ^{2} \frac{k_{y}}{2}}
$$

where $\epsilon^{a, b}=\frac{K_{\perp}^{a, b}}{K_{\|}}$are the relative interchain elastic couplings. The equations governing the classical fields $\phi_{0}^{\mathbf{j}}$ now become:

$$
\begin{aligned}
\partial_{x}^{2} \phi_{0}^{\mathbf{j}} & +\frac{1}{2 \xi^{2}} \mathbf{B}(\mathbf{0}) \sin \left(2 \phi_{0}^{\mathbf{j}}\right) \\
& +\frac{1}{\xi^{2}} \sum_{\mathbf{j}^{\prime} \neq \mathbf{j}} \mathbf{B}\left(\mathbf{j}^{\prime}-\mathbf{j}\right) \cos \phi_{0}^{\mathbf{j}} \sin \phi_{0}^{\mathbf{j}^{\prime}}=0
\end{aligned}
$$

where we have only included for simplicity the effect of the NN exchange interaction in each chain. In this situation, $\xi$ is given by Eq. (9) with $g=0$. Moreover, as the interchain elastic couplings do not affect the uniform dimerized state, the relation (11) between $\xi$ and the gap is preserved.

The second term of Eq. (17) is dominant due to the rapidly decaying effective interaction $\mathbf{B}\left(\mathbf{j}^{\prime}-\mathbf{j}\right)$ with the distance $\left|\mathbf{j}^{\prime}-\mathbf{j}\right|$. This behavior can be seen in Fig. 2 for the case $\epsilon^{a}=\epsilon^{b}$. By neglecting the last term in Eq. (17) we obtain a system of decoupled equations of the form (7). We consider a situation where a soliton is formed in a single chain in an otherwise SP state. Therefore we have $\phi_{0}^{\mathbf{j}}=\pi / 2$ for $\mathbf{j} \neq \mathbf{m}$ and $\sin \left(\phi_{0}^{\mathbf{j}}\right)=\tanh \left(x / \xi^{\prime}\right)$ for $\mathbf{j}=\mathbf{m}$. The lattice pattern is obtained from Eq. (15):

$$
u^{\mathbf{j}}=\mathbf{B}(\mathbf{j}-\mathbf{m}) u_{0} \tanh \left(x / \xi^{\prime}\right)+(1-\mathbf{B}(\mathbf{j}-\mathbf{m})) u_{0}
$$

This solution represents a soliton at the origin of the $\mathbf{m}$ chain. It is easy to see that the displacement amplitude at one side of the soliton is smaller than at the other side. This difference can be explained by the fact that at one side the dimerization has the same phase with respect to that of the neighboring chains while at the other these phases are opposite. At the same time, the neighbor chains $\mathbf{j} \neq \mathbf{m}$ feel the presence of the soliton in $\mathbf{m}$. As seen from Eq. (18) they are slightly distorted from the uniform dimerized pattern.

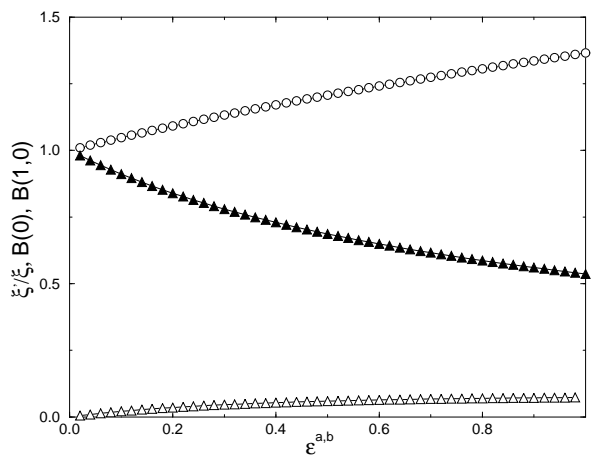

FIG. 2. The ratio between the effective soliton width and the "bare" soliton width as a function of $\epsilon^{a}=\epsilon^{b}$ (circles). Coefficients $B(\mathbf{0})$ (filled triangles) and $B(1,0)$ (open triangles) given by Eq. (16) are also shown.

From Eq. (17) we obtain the effective soliton width $\xi^{\prime}=\xi / \sqrt{B(\mathbf{0})}$ which is larger than the "bare" width $\xi$ of the one chain problem previously discussed since $B(\mathbf{0})<1$. We show in Fig. 22 the behavior of $\xi^{\prime} / \xi$ as a function of $\epsilon^{a}$ for the case of $\epsilon^{a}=\epsilon^{b}$. It is difficult to extract from experimental data an estimation of the values of $\epsilon^{a, b}$ since these are effective elastic constants as introduced in the interaction term (14).

After this work has been-gompleted we became aware of a paper of Zang et al.17 where the effects of frustration and interchain magnetic coupling on the soliton width were studied. Their conclusion about the frustration effects is similar to the one presented here. Besides, they conclude that the large soliton width experimentally found in $\mathrm{CuGeO}_{3}$ is mainly due to the antiferromagnetic interchain coupling. However, as the study of Ref. [17] is based on a mean-field approximation for the interchain coupling and since the soliton formation is a strongly local phenomena, we think that the enhancement of the soliton width found by these authors might be overestimated. In this case, a contribution from the interchain elastic coupling could be needed to explain the experimental result. The question of to what extent these two mechanisms contribute to the soliton width could only be answered by studying a model which includes both magnetic and elastic interchain couplings.

${ }^{1}$ M. Hase, I. Terasaki and K. Uchinokura, Phys. Rev. Lett. 70, 3651 (1993).

2 J.-L. Lussier, S. M. Coad, D. F. McMorrow, and D. McK. Paul, J. Phys. Condens. Matter 7, 325 (1995).

${ }^{3}$ J. Riera and A. Dobry, Phys. Rev. B 51, 16098 (1995).

${ }^{4}$ G. Castilla, S. Chakravarty and V.J. Emery, Phys. Rev. Lett. 75, 1823 (1995).

${ }^{5}$ S. Haas and E. Dagotto, Phys. Rev. B 52, 14396 (1995).

${ }^{6}$ J. Riera and S. Koval, Phys. Rev. B 53, 770 (1996).

${ }^{7}$ D. Poilblanc et al., Phys. Rev. B, to appear (1997).

${ }^{8}$ V. Kiryukhin, B. Keimer, J. Hill, and A. Vigliante, Phys. Rev. Lett. 76, 4608 (1996); V. Kiryukhin et al., Phys. Rev. B 54, 7269 (1996).

${ }^{9}$ T. Nakano and H. Fukuyama, J. Phys. Jpn 49, 1679 (1980).

${ }^{10}$ J.E. Lorenzo et al., Phys. Rev. B 50, 1278 (1994).

${ }^{11}$ K. Hirota et al., Phys. Rev. Lett. 73, 736 (1994).

12 M. Saint-Paul et al., Phys. Rev. B 55, 6121 (1997).

${ }^{13}$ I. Affleck, Fields Strings and Critical Phenomena, edited by E. Brézin and J.Zinn-Justin (North-Holland, Amsterdam 1990), pg. 563.

14 J. Zang, A.R. Bishop and D. Schmeltzer, Phys. Rev. B 52, 6723 (1995); 549556 (1996). Note however that our choice of the boson field $\phi$ is the same as in Ref. [9]. 
${ }^{15}$ A. Feiguin, A. Dobry, J. Riera, and A. Ceccatto, (to be published).

16 A. Fledderjohann and C. Gros, cond-mat/9612013.

17 J. Zang, S. Chakravarty and A.R. Bishop, condmat/9702185. 